\title{
Relation température-profil d'émission dans la luminescence de l'exciplexe $\mathrm{Kr}^{*}$ Ar
}

\author{
J.-L. Subtil, C. Vincent-Donnet, P. Laporte, R. Reininger* et P. Gürtler**
}

Laboratoire TSI, URA 842, Université Jean Monnet, 23 rue du Dr P. Michelon, 42023 Saint-Etienne, France

* Synchrotron Radiation Center, University of Madison Wisconsin, 3731 Schneider Dr, Stoughton WI 53581, U.S.A.

** HASYLAB/DESY, Notkestrasse 85, 2000 Hamburg 52, Germany

\begin{abstract}
The fast $0^{+}\left({ }^{3} \mathrm{P}_{1}\right)$ and slow $1\left({ }^{3} \mathrm{P}_{2}\right)$ relaxed exciplex $\mathrm{Kr}{ }^{*} \mathrm{Ar}$ emissions near $9.26 \mathrm{eV}(134 \mathrm{~nm})$ have been observed in a large density and temperature range. The band half widths are found to be only temperature dependent, a result similar to that previously reported for the rare gas excimers (homonuclear), and revealing a very week environmental influence. From a simple model we derive $\omega_{\mathrm{e}}=60 \mathrm{~cm}^{-1}$ for the vibrational constant and $\Delta_{0}=2400 \mathrm{~cm}^{-1}$ for the width extrapolated at $\mathrm{O}$ Kelvin.
\end{abstract}

Différents travaux antérieurs sur les gaz rares purs ont montré la très faible influence de l'environnement sur le profil d'émission de l'excimère dans l'ultra-violet du vide. Jortner et al. ${ }^{1}$ ont en particulier mis en évidence que la largeur de la bande ne dépendait pratiquement que de la température avec, notamment, un effet négligeable dans le changement de phase gaz/liquide à température quasi constante (argon, xénon).

Ces résultats ont pu être interprétés dans une perspective purement moléculaire avec un modèle simple de potentiel harmonique. Plus récemment, plusieurs d'entre nous ${ }^{2}$ ont repris ces travaux d'une part en les étendant au krypton, et d'autre part en étudiant l'ensemble du domaine de densité s'étendant du gaz au liquuide ce qui implique le contournement du point critique par une maîtrise appropriée des conditions thermodynamiques.

Le présent travail concerne les premiers résultats équivalents, jamais obtenus dans le cas des exciplexes gaz rare - gaz rare. La paire étudiée est le couple $\mathrm{Kr}^{*}$ Ar pour lequel l'émission se situe à $134 \mathrm{~nm}$. L'observation porte sur l'ensemble du domaine gaz - liquide et aussi bien sur la composante dite rapide (transition $\left.\mathrm{O}^{+}\left({ }^{1} \mathrm{~S}_{0}\right) \leftarrow \mathrm{O}^{+}\left({ }^{3} \mathrm{P}_{1}\right)\right)$ que sur celle dite lente (transition $\left.\mathrm{O}^{+}\left({ }^{1} \mathrm{~S}_{0}\right) \leftarrow 1\left({ }^{3} \mathrm{P}_{2}\right)\right)$. 
Les profils ont pu être obtenus dans de très bonnes conditions expérimentales mettant en oeuvre le rayonnement synchrotron (DESY / Hambourg) et des échantillons à dilution extrême ( $\approx 1 \mathrm{ppm}$ de krypton dans l'argon). Un effet notable de la temperature mais de comportement assez différent du cas des gaz rares purs est apparent (fig. 1). Ceci est vraisemblablement dû à la nature des potentiels associés à l'exciplexe $\mathrm{Kr}^{*} \mathrm{Ar}$ qui sont beaucoup moins profonds que dans les cas précédents ${ }^{3}$. A la précision de mesure près, les largeurs associées aux composantes lentes et rapides sont trouvées identiques, ce qui est consistant avec les potentiels théoriques ${ }^{3}$. Le modèle de Jortner et al $^{1}$ a été appliqué aux résultats expérimentaux. L'expression de la largeur de la bande émise $\Delta$ est la suivante :

$$
\Delta=\Delta_{0}\left[\operatorname{coth}\left(\frac{\hbar \omega_{\mathrm{e}}}{2 \mathrm{k}_{\mathrm{B}} \mathrm{T}}\right)\right]^{1 / 2}
$$

Avec $\Delta_{0}$ : largeur à mi-hauteur extrapolée à température nulle et $\omega_{\mathrm{e}}$ : constante vibrationnelle. L'estimation, commune pour les deux états exciplexes conduit à $\omega_{\mathrm{e}}=60 \mathrm{~cm}^{-1}$ et $\Delta_{0}=2400 \mathrm{~cm}^{-1}$.

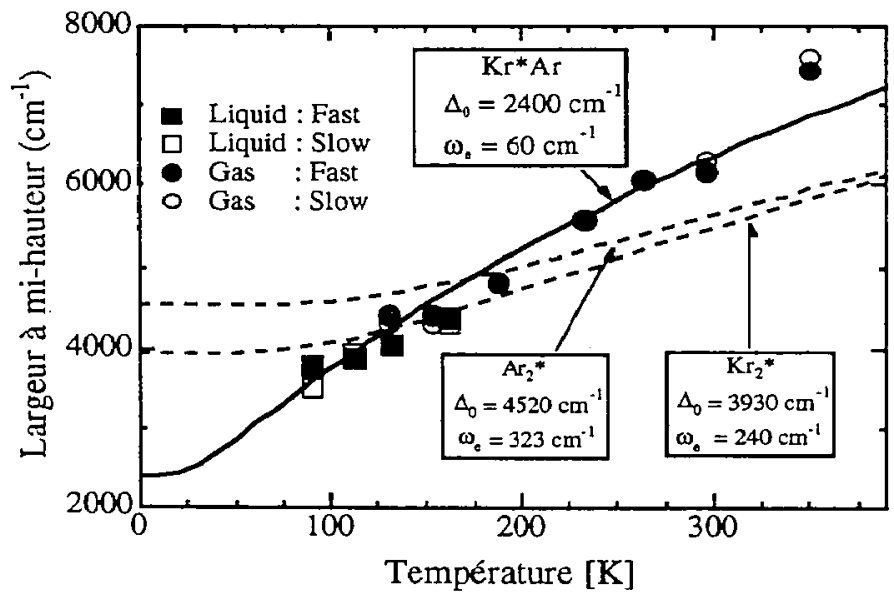

Figure 1 : Influence de la température sur la largeur à mi-hauteur de l'émission de l'exciplexe $\mathrm{Kr} r^{*} \mathrm{Ar}$ à $134 \mathrm{~nm}$. Les données correspondantes de $\mathrm{Ar}_{2}{ }^{*}$ et de $\mathrm{Kr}_{2}{ }^{*}$ sont tirées de la référence 2.

Les potentiels $\mathrm{Kr}^{*} \mathrm{Ar}$ ont été calculés théoriquement récemment ${ }^{3}$ ce qui permettra une interprétation plus poussée dans les développements en cours actuellement.

Les auteurs remercient l'OTAN pour sa participation financière à la présente recherche (contrat $n^{\circ}$ CRG 920574).

(1) O. Cheshnovsky, B. Raz and J. Jortner Chem. Phys. Lett. 475, 15 (1972)

(2) E. Morikawa, R. Reininger, P. Gürtler, V. Saile and P. Laporte J. Chem. Phys. J469, 91 (1989)

(3) F. Spiegelmann, F.X. Gadéa and M.C. Castex Chem. Phys. 173, 145 (1990) 